\title{
Worksite back and core exercise in firefighters: Effect on development of lumbar multifidus muscle size
}

\author{
John M. Mayer* and James L. Nuzzo \\ School of Physical Therapy and Rehabilitation Sciences, Morsani College of Medicine, University of South \\ Florida, Tampa, FL, USA
}

Received 4 January 2013

Accepted 9 October 2013

\begin{abstract}
. on back muscle size and symmetry has not been investigated in firefighters. muscle hypertrophy in firefighters. mass) L4 and L5 lumbar multifidus muscle CSA and asymmetry values. the lumbar multifidus muscle in healthy firefighters.

Keywords: Muscles, hypertrophy, exercise training, spine, ultrasonography

\section{Introduction}

Firefighting is a dangerous occupation with a high incidence of low back pain and injury [1]. In a recent report, $86 \%$ of career firefighters reported a history of low back pain, while $55 \%$ reported current low back pain [1]. Back injury is also the leading cause of early retirement in firefighters [1]. While the causes of low back pain and injury in firefighters remain unclear,
\end{abstract}

BACKGROUND: Firefighting is a dangerous occupation with a high incidence of low back pain and injury. Abnormal back muscle function and morphology has been linked to low back pain and poor physical performance. The effect of exercise training

OBJECTIVE: The purpose of this study was to assess the effect of worksite exercise training for eliciting lumbar multifidus

METHODS: A cluster randomized controlled trial was conducted with healthy, career firefighters $(n=64)$ from a medium-sized fire department. Participants were randomized by fire station to exercise training $(n=36)$ (supervised back and core exercise performed on duty, 2X/week, 24 weeks) or control ( $n=28$ ). The cross-sectional area (CSA) of the L4 and L5 lumbar multifidus muscle was assessed with ultrasonography at baseline and following the intervention.

RESULTS: At 24 weeks, no significant differences were noted between the groups in the adjusted (by baseline scores and body

CONCLUSIONS: A worksite exercise training program targeting the back and core is not effective for eliciting hypertrophy of

${ }^{*}$ Corresponding author: John M. Mayer, School of Physical Therapy and Rehabilitation Sciences, Morsani College of Medicine, University of South Florida, 12901 Bruce B. Downs Blvd, MDC77, Tampa, FL 33612, USA. Tel.: +1 813974 3818; Fax: +1 813974 8915; E-mail: jmayer2@health.usf.edu. multiple factors likely contribute to the high incidence of low back pain and injury in firefighters. Preliminary studies suggest that various physical firefighting activities (e.g., operating charged hoses, climbing ladders, breaking windows, looking for hidden fires, and lifting objects $\geqslant 18 \mathrm{~kg}$ ) and awkward lifting postures are linked with low back injury [23]. Furthermore, aberrant musculoskeletal movement patterns have been associated with musculoskeletal injuries in firefighters at the training academy [4].

In non-firefighter populations, atrophy of the lumbar multifidus has also been associated with low back pain [5-7]. For example, Hides et al. [6] found lumbar multifidus cross-sectional areas (CSA) at the L4 and L5 levels to be significantly smaller in individuals with 
low back pain compared to asymptomatic individuals. Consequently, many investigations have sought to determine if exercise interventions are effective for eliciting multifidus hypertrophy [8-14]. In non-firefighters with low back pain [8]10|11|13] and in post-operative patients [9], various rehabilitative exercise interventions have been demonstrated to be effective at eliciting multifidus hypertrophy. However, less research has assessed the effectiveness of preventative exercise interventions for eliciting multifidus hypertrophy in healthy individuals [12 14], and no data are available in firefighters.

Due to the high incidence of low back pain and injury in firefighters, and the association between low back pain and multifidus atrophy, identifying exercise interventions that elicit multifidus hypertrophy in firefighters appears warranted and potentially valuable to the fire service and other high-risk occupations. Thus, the purpose of this study was to assess the effect of a worksite exercise training program for eliciting lumbar multifidus muscle hypertrophy in firefighters.

\section{Methods}

\subsection{Study design}

This study was a two-arm, cluster randomized controlled trial. Participants were randomized by fire station into either an exercise training group ( 24 weeks of supervised exercise training at the fire station) or a control group. The outcome measures (multifidus CSA and asymmetry) were collected at a university laboratory at baseline and after 24 weeks. Data were collected from February 2011 through November 2011.

\subsection{Participants}

Participants ( $n=64,6$ female, 58 male) were career firefighters who were recruited from the entire population of firefighters of Tampa Fire Rescue ( $n=$ 573) (Tampa, FL, USA). Methods of recruitment included email notifications, presentations and posted flyers at the fire stations, and word-of-mouth. Eligibility of study candidates was assessed with a brief telephone screen, which included questions about work status and health history. Eligible candidates were then invited to the university's laboratory for additional onsite screening, which included various self-reported health questionnaires, urine pregnancy test for females, and a physical examination by a medical physician. In- formed consent was obtained from all study candidates prior to participation in the on-site screening procedures. The study protocol was approved by the university's institutional review board.

To be included in the study, participants had to be $\geqslant$ 18 years of age and an active, full-duty firefighters of Tampa Fire Rescue without work restrictions. Exclusion criteria for the study were as follows: cardiovascular or orthopaedic contraindications to resistance exercise [15]; history of systemic inflammatory disease or spinal surgery; clinically significant self-reported current low back pain or disability [1617]; level of readiness for physical activity at screening deemed by the study physician to preclude participation [18]; presence of a red flag for potentially serious condition related to low back pain [19]; resting blood pressure or heart rate outside of normal limits at screen (normal limits: blood pressure systolic 90-139 mm Hg, diastolic $60-89 \mathrm{~mm} \mathrm{Hg}$; heart rate $60-100$ beats per minute for sedentary individuals); currently receiving care for or disabled due to spinal pain disorder or injury; currently diagnosed with or receiving treatment for a psychological or psychiatric disorder; currently performing progressive resistance exercises for the low back or core muscles; active workers' compensation or personal injury case; unable or unwilling to complete the study procedures; research team member (e.g. peer fitness trainer); pregnant; simultaneously enrolled in another biomedical clinical trial; drug or alcohol abuse within the past year; or any other condition, which in the opinion of the principal investigator or study physician, would put the candidate at increased safety risk or otherwise make the candidate unsuitable for this study.

\subsection{Randomization}

The unit of randomization for this study was fire station. The purpose of randomizing by cluster (fire station) rather than individual was to help minimize the potential of exposure bias and contamination. Since firefighters at a given fire station are in each other's company for 24 hours at a time, randomizing by individual would likely have created opportunities for firefighters in one study group to discuss the study with firefighters in the other study group and to observe the other group's exercise training.

Prior to enrollment, the statistician stratified the fire stations of Tampa Fire Rescue by certain characteristics (number of firefighters, age of firefighters, number of emergency responses), and fire stations with similar characteristics were paired. Using a statisti- 
cal software program (Statistical Analysis System, version 9.2, Cary, NC, USA), the statistician generated a randomization sequence for each pair of fire stations. Based on the randomization scheme, each fire station had an equal chance of being assigned to either the exercise or control group. Group assignment was revealed to the investigators and study participants after all baseline assessments were completed in a pair of stations. The statistician, study physician, and radiologist who conducted the image analysis were blinded to group assignment throughout the study.

\subsection{Intervention}

\subsubsection{Exercise}

Within approximately one week after randomization, individuals in the exercise training group initiated participation in the supervised exercise program at the fire stations. The participants completed the exercise program everyday while on duty over the course of 24 weeks (approximately two times per week based on the normal shift schedule for firefighters at Tampa Fire Rescue: 24 hours on duty, followed by 48 hours off duty, with an extra day off every three weeks ("R" day)). For each exercise session, five exercises were completed: four core exercises on an exercise mat and one back extension exercise on a variable angle Roman chair. The total time to complete the five exercises was approximately 10 to 15 minutes. The exercises and progression protocol used in the current study have been found to activate the back and core muscles, and improve back and core muscle endurance in nonfirefighters [20-22].

\subsubsection{Core exercises}

To begin each exercise session, the following core exercises were completed: Cat Camel, Birddog, Curlup, and Side Bridge [21]. The Cat Camel involved the following techniques: quadruped start position; cycling through full spinal flexion and extension; maintaining isometric contractions of six to eight seconds at the maximum spinal flexion and extension positions. The Birddog involved the following techniques: quadruped start position; simultaneous lifting of the contralateral upper and lower limbs to be parallel with the floor; maintaining an isometric contraction of six to eight seconds at the parallel position. The Curl-up involved the following techniques: supine position start position, with one knee flexed, hands placed under the low back, and elbows in contact with the mat; lifting the head and shoulders up toward the ceiling as high as possible; maintaining an isometric contraction of six to eight seconds at this position. The Side Bridge involved the following techniques: side-lying start position, with knees bent at $90^{\circ}$ and one elbow bent at $90^{\circ}$ and positioned under the shoulder; lifting the pelvis to a position of $0^{\circ}$ hip flexion, with only the knees and elbows in contact with the mat; maintaining an isometric contraction of six to eight seconds at this position.

One set of five repetitions was completed for each core exercise. For the Birddog, five repetitions were performed with the right leg and left arm, and five repetitions were performed with the left leg and right arm. For the Side Bridge, five repetitions were performed on the right side, followed by five repetitions on the left side. No rest was provided between the four core exercises. The volume and intensity of the core exercises remained constant throughout the 24 -week training period.

\subsubsection{Back extension exercise}

Following the core exercises, the participant completed one set of back extension using a variable angle Roman chair (Conner Athletic Products, Jefferson, Iowa, United States). The variable angle Roman chair was fixed at one of six angles $\left(75^{\circ}, 60^{\circ}, 45^{\circ}, 30^{\circ}, 15^{\circ}\right.$ or $0^{\circ}$ during the set of exercise. Standard positioning of the participant on the variable angle Roman chair included the following: aligning the anterior superior iliac spines with the superior edge of the machine's pelvic restraint pad; securing the ankles under the machine's ankle pad; extending the knees fully; and internally rotating the hips such that the toes point inward at $45^{\circ}$ [23].

Each repetition took 12 seconds to complete and consisted of four-second eccentric, concentric, and isometric contractions. To standardize the movement velocity, a metronome (MA-30, Korg Inc., Tokyo, Japan) was used during the set. The participant started each repetition in terminal extension. Then, over a period of four seconds, the participant lowered the torso in a smooth, controlled manner to terminal flexion. Next, the participant lifted the torso to terminal extension over a period of four seconds. Finally, the participant held the terminal extension position for a period of four seconds, prior to starting the eccentric phase of the next repetition. Throughout the exercise set, the participant was instructed to accentuate lumbar intersegmental extension and to limit movement at the hip and knee joints [23]. The participant performed the set of exercise until volitional fatigue or 30 repetitions were attained. The goal repetition range was $15-20$ at vo- 


\begin{tabular}{|c|c|c|}
\hline Machine Angle & Hand Position & Resistance \\
\hline $75^{\circ}$ & $\begin{array}{l}\text { 1. Hands on Sternum } \\
\text { 2. Genie Arm Cross }\end{array}$ & $\begin{array}{l}\text { LEAST } \\
\text { (easiest) }\end{array}$ \\
\hline $60^{\circ}$ & $\begin{array}{l}\text { 4. Hands on Sternum } \\
\text { 5. Genie Arr Cross } \\
\text { 6. Behind the Head }\end{array}$ & \\
\hline $45^{\circ}$ & $\begin{array}{l}\text { 7. Hands son Sternum } \\
\text { 8. Genie Arm Cross } \\
\text { 9. Behind the Head }\end{array}$ & \\
\hline $30^{\circ}$ & $\begin{array}{l}\text { 10. Hands on Sternum } \\
\text { 11. Genie Arm Cross } \\
\text { 12. Behind the Head }\end{array}$ & \\
\hline $15^{\circ}$ & $\begin{array}{l}\text { 13. Hands on Sternum } \\
\text { 14. Genie Arm Cross } \\
\text { 15. Behind the Head }\end{array}$ & \\
\hline $\begin{array}{ll}-9 & 0 \\
& 0\end{array}$ & $\begin{array}{l}\text { 16. Hands on Sternum } \\
\text { 17. Genie Arr Cross } \\
\text { 18. Behind the Head }\end{array}$ & $\begin{array}{l}\text { MOST } \\
\text { (hardest) }\end{array}$ \\
\hline
\end{tabular}

Fig. 1. Progressive resistance exercise protocol used for back extension exercise training.

litional fatigue. Over the course of the 24-week training period, the resistance level was regularly altered for subsequent exercise sessions. The resistance level was altered by adjusting the variable angle Roman chair angle setting (six settings) and/or by manipulating the participant hand position (three hand positions) [20] (Fig. 1). External weights were not used. By altering the angular position of the exercise device and/or participant hand position, the resistive load applied to the low back region is changed based on the action of gravity on the upper body. A similar standardized progression protocol was used in a previous exercise training study that enrolled healthy non-firefighter adults [22]. For the first session of exercise training, each participant completed the back extension exercise at the lowest resistance level. The resistance level used in subsequent exercise sessions was based on performance: $\geqslant 20$ repetitions at one session or 15-19 repetitions at three consecutive sessions - increase resistance by one level; $\leqslant 14$ repetitions at three consecutive sessions decrease resistance by one level.

\subsubsection{Exercise supervision}

A certified exercise trainer supervised every exercise session over the course of the 24 -week program. The exercise trainers consisted of an exercise specialist from the research team and peer fitness trainers [1] from the fire department. Although the exercise sessions were supervised, they were not completed at the same time every day. This was because the participants were unable to suspend their usual fire service activities (e.g., emergency responses) in order to perform the exercises on duty. Thus, the exercise trainer worked around the daily operations of each fire station.

\subsubsection{Control}

Participants in the control group did not receive the supervised intervention. Participants in both the con-

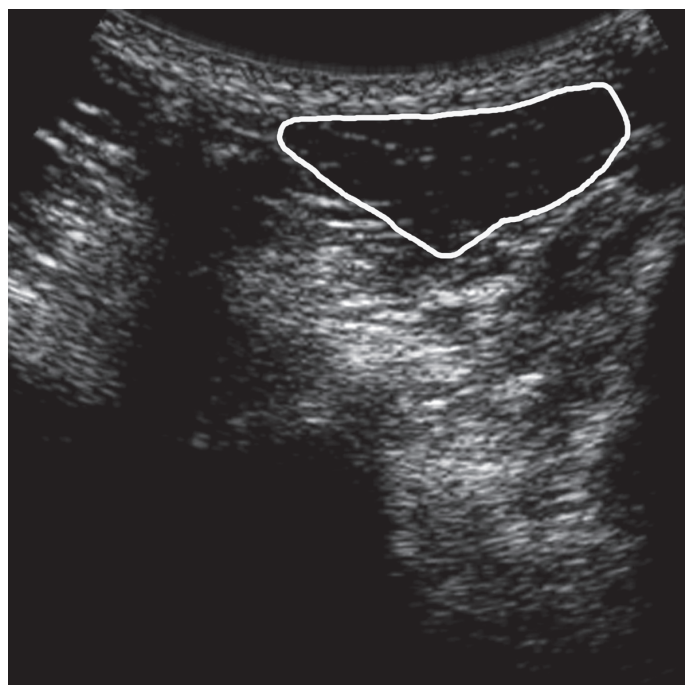

Fig. 2. Cross-sectional ultrasound image of L5 lumbar multifidus $(\mathrm{MU}) . \mathrm{LA}=$ lamina; $\mathrm{SP}=$ spinous process.

trol group and exercise group were given verbal and written instructions to continue performing any usual physical fitness routines throughout the 24-week study period, not start any new exercises for the back and core muscles, and not perform any progressive resistance exercise for the back muscles. General questioning at the follow-up study visits was used to monitor compliance with these instructions.

\subsection{Ultrasonography}

The primary outcome measures for this study were lumbar multifidus CSA and asymmetry, which were assessed at baseline and at 24 weeks following the onset of the intervention. Lumbar multifidus CSA images were acquired with a portable ultrasound device (MyLab 25, Biosound Esaote Inc., Florence, Italy), which was equipped with a $5 \mathrm{MHz}$ curvilinear transducer (CA631, Biosound Esaote Inc., Florence, Italy). Images were acquired from the participants' left and right sides at the L4 and L5 vertebral levels. During image acquisition, participants laid prone on a table, with a pillow underneath the abdomen to standardize the position of lordosis [11]. To standardize the transducer position for image acquisition, the test examiner palpated bony landmarks and then identified the landmarks on the device's monitor.

The images were analyzed on a desktop computer using ImageJ software (National Institute of Health, Bethesda, Maryland, United States). The ImageJ freehand tool was used to trace the borders of the lum- 
Table 1

Baseline characteristics of participants

\begin{tabular}{lccc}
\hline & $\begin{array}{c}\text { Exercise }(n=36) \\
\text { Mean } \pm \text { SD }\end{array}$ & $\begin{array}{c}\text { Control }(n=28) \\
\text { Mean } \pm \text { SD }\end{array}$ & $\begin{array}{c}\text { Total }(n=64) \\
\text { Mean } \pm \text { SD }\end{array}$ \\
\hline Age $(\mathrm{y})$ & $36.1 \pm 10.0$ & $31.8 \pm 8.5$ & $34.2 \pm 9.6$ \\
Career as firefighter $(\mathrm{y})$ & $9.6 \pm 9.4$ & $6.6 \pm 7.6$ & $8.3 \pm 8.7$ \\
Any physical exercise $(\mathrm{d} / \mathrm{wk})$ & $3.0 \pm 1.3$ & $3.7 \pm 1.5$ & $3.3 \pm 1.4$ \\
Body Height $(\mathrm{cm})$ & $176.7 \pm 8.2$ & $177.5 \pm 10.1$ & $177.1 \pm 9 . .0$ \\
Body Mass $(\mathrm{kg})$ & $86.5 \pm 13.1$ & $85.4 \pm 19.1$ & $86.0 \pm 15.9$ \\
BMI $\left(\mathrm{kg} / \mathrm{m}^{2}\right)$ & $27.7 \pm 3.6$ & $26.9 \pm 4.2$ & $27.3 \pm 3.9$ \\
Body fat $(\%)$ & $23.1 \pm 10.1$ & $20.5 \pm 6.2$ & $22.0 \pm 8.6$ \\
\hline
\end{tabular}

$\mathrm{BMI}=$ body mass index; $\mathrm{SD}=$ standard deviation.

Table 2

Unadjusted lumbar multifidus muscle cross-sectional area and asymmetry values at baseline and 24-weeks

\begin{tabular}{lccccc}
\hline & \multicolumn{2}{c}{ Exercise $(n=36)$} & & \multicolumn{2}{c}{ Control $(n=28)$} \\
\cline { 2 - 3 } \cline { 5 - 6 } Variable & Baseline & 24-weeks & & Baseline & 24 -weeks \\
\hline Right L4 CSA $\left(\mathrm{cm}^{2}\right)$ & $11.2 \pm 2.2$ & $10.7 \pm 1.5$ & & $11.0 \pm 2.5$ & $10.7 \pm 1.7$ \\
Left L4 CSA $\left(\mathrm{cm}^{2}\right)$ & $12.0 \pm 2.7$ & $11.3 \pm 1.8$ & & $11.3 \pm 2.5$ & $11.0 \pm 1.9$ \\
Right L5 CSA $\left(\mathrm{cm}^{2}\right)$ & $11.3 \pm 2.1$ & $10.2 \pm 1.6$ & & $11.1 \pm 2.3$ & $10.1 \pm 1.9$ \\
Left L5 CSA $\left(\mathrm{cm}^{2}\right)$ & $11.2 \pm 1.9$ & $10.3 \pm 1.8$ & & $11.0 \pm 2.4$ & $10.3 \pm 1.9$ \\
L4 asymmetry (\%) & $9.8 \pm 9.3$ & $9.6 \pm 7.7$ & & $7.2 \pm 6.7$ & $9.0 \pm 6.5$ \\
L5 asymmetry (\%) & $7.7 \pm 6.8$ & $6.6 \pm 5.9$ & & $11.6 \pm 21.4$ & $7.4 \pm 6.5$ \\
\hline
\end{tabular}

$\mathrm{CSA}=$ cross-sectional area. Values are reported as mean \pm standard deviation.

bar multifidus [11] (Fig. 2). The echogenic lamina was used to identify the multifidus deep border, the acoustic shadow of the spinous process was used to identify the multifidus medial border, the thoracolumbar fascia was used to identify the multifidus superficial border, and the fascia separating the lumbar multifidus from the erector spinae was used to identify the multifidus lateral border. The mean value of two measurements, which came from two separate ultrasound images, was used for the final analysis. Asymmetry between right and left lumbar multifidus CSA was expressed as the percent difference between sides (\% difference $=[($ largest $/$ smallest value $) \times 100]-100)[24]$. Ultrasonography is a reliable and valid tool for assessing lumbar multifidus CSA [25].

\subsection{Statistical analyses}

For the primary outcome measures (multifidus CSA and asymmetry), the research aim was to determine if a difference existed between the exercise and control groups at the 24-week time point. Analysis of covariance (ANCOVA), which controlled for baseline scores of the outcome measures and body mass, was used to assess group differences at 24 weeks for each outcome measure. Intention to treat analysis was used and statistical significance was accepted with alpha set at 0.05 . Data are reported as mean \pm standard deviation (SD), unless otherwise noted. All analyses were con- ducted using SPSS version 20 (IBM, Armonk, New York, United States).

\section{Results}

\subsection{Participant characteristics}

Participant characteristics are shown in Table 1. A total of 64 firefighters from 15 fire stations completed the study's end of trial assessments (exercise: $n=36$ firefighters from 8 stations; control: $n=28$ firefighters from 7 stations).

\subsection{Exercise training characteristics}

The mean \pm SD number of exercise training sessions completed over the 24-week training period by each participant in the exercise group was $33.3 \pm$ 8.7 , which was approximately $69 \%$ of the target of 48 exercise sessions. Reasons for missed exercise training sessions included: did not temporarily meet inclusion/exclusion criteria at the time of presenting for a particular exercise training session, elected not to participate in a particular training session, leave (e.g. annual, personal, sick), other/unknown, out of station (e.g. emergency response, mandatory training, miscellaneous), no exercise trainer available to supervise an exercise session during a scheduled on-duty day for the participant, planned rest day prior to study assessment visit, and transfer to a control station. 
Table 3

Adjusted* lumbar multifidus muscle cross-sectional area and asymmetry values at 24 -weeks

\begin{tabular}{lcc}
\hline Variable & $\begin{array}{c}\text { Exercise }(n=36) \\
\text { Mean } \pm \mathrm{SE}\end{array}$ & $\begin{array}{c}\text { Control }(n=28) \\
\text { Mean } \pm \mathrm{SE}\end{array}$ \\
\hline Right L4 CSA $\left(\mathrm{cm}^{2}\right)$ & $11.2 \pm 0.3$ & $11.0 \pm 0.3$ \\
Left L4 CSA $\left(\mathrm{cm}^{2}\right)$ & $10.6 \pm 0.2$ & $10.7 \pm 0.3$ \\
Right L5 CSA $\left(\mathrm{cm}^{2}\right)$ & $10.2 \pm 0.3$ & $10.2 \pm 0.3$ \\
Left L5 CSA $\left(\mathrm{cm}^{2}\right)$ & $10.3 \pm 0.3$ & $10.3 \pm 0.3$ \\
L4 asymmetry (\%) & $9.7 \pm 1.2$ & $9.2 \pm 1.4$ \\
L5 asymmetry $(\%)$ & $6.3 \pm 1.1$ & $7.7 \pm 1.2$ \\
\hline
\end{tabular}

CSA $=$ cross-sectional area; $\mathrm{SD}=$ standard error. $*$ Adjusted for baseline score and body mass.

\subsection{Compliance and safety}

No participant reported performing any other exercises for the back or core outside of those assigned for the study. No related or possibly related serious adverse events were observed. Related and possibly related discomforts were generally consistent with typical responses to resistance exercise (e.g. temporary soreness and stiffness), minor, self-limiting, and did not impact work status.

\subsection{Primary outcomes - lumbar multifidus muscle CSA and asymmetry}

Raw unadjusted lumbar multifidus muscle CSA and asymmetry values at baseline and 24 weeks are found in Table 2. Based on the ANCOVA results, no significant differences between the exercise and control groups were observed for any of the adjusted lumbar multifidus CSA and asymmetry values (Table 3).

\section{Discussion}

The current study is the first to assess the efficacy of a worksite exercise training program for eliciting lumbar multifidus muscle hypertrophy in firefighters. The primary finding from the current study was that the supervised worksite exercise program was not effective at eliciting lumbar multifidus hypertrophy. If lumbar multifidus hypertrophy is a goal in firefighter wellness and fitness programs aimed at healthy firefighters, then the specific exercise program assessed in the current study is not advised.

Various factors may explain why the exercise intervention in the current study was not effective at eliciting lumbar multifidus hypertrophy. First, it is possible that the baseline multifidus CSA was high compared with reference populations. For example, the mean values of multifidus CSA measurements of the firefighters in the current study appeared to be greater than mean values for the general population [24]. However, the mean values for firefighter multifidus CSA fell within the $95 \%$ reference ranges for the general population [24]. Second, the mode, volume (sets and repetitions), or intensity (resistance) of exercise may not have been ideal for eliciting hypertrophy. Similar to the current study findings, Teyhen et al. [14] did not find significant improvements in lumbar multifidus thickness in healthy soldiers who participated in a 12week, low intensity, non-progressive exercise program consisting of either traditional exercises (e.g., sit-ups, crunches) or core stabilization exercises (e.g., abdominal drawing-in maneuver, quadruped alternate arm and leg). On the contrary, Kim et al. [12] found significant improvements in the size of the lumbar multifidus and other lumbar paraspinal muscles in healthy adults following an 8-week machine-based progressive resistance exercise program using a higher exercise intensity with external loads equaling $70 \%$ of onerepetition maximum. Thus, higher exercise intensities higher than those administered in the current study may be necessary to elicit multifidus hypertrophy in healthy adults.

A limitation to the current study was that only firefighters without current low back pain participated. Thus, the findings of the present study are not generalizable to firefighters with existing multifidus atrophy and low back pain, and to non-firefighter populations. Also, the current study involved very specific exercise modes, volumes, and intensities of exercise. Furthermore, based on the results of this study, it is unknown if the exercise training program results in benefits such as increased self-reported energy levels, and improvements in materials handling, work performance, and activities of daily living.

Future research is needed to determine if the studied exercise intervention may be effective at eliciting hypertrophy in firefighters with muscle atrophy and low back pain. Future research is also required to determine if different types of exercise interventions are better suited for eliciting multifidus hypertrophy in firefighters. Additionally, future research is needed to assess the relationship between lumbar multifidus size, clinical outcomes, such as incidence of low back injury, and occupational outcomes, such as job performance and lost work days.

\section{Conclusion}

A 24-week supervised worksite exercise program, which consisted of core stability and progressive re- 
sistance back extension exercise, was not effective for eliciting hypertrophy of the lumbar multifidus muscle in healthy firefighters. Further research is required to assess the clinical implications of these findings and the impact of other exercise approaches on lumbar multifidus muscle CSA.

\section{Acknowledgements}

This study was funded by a Fire Prevention and Safety Grant (Number: EMW-2009-FP-00418) from the Federal Emergency Management Agency, U.S. Department of Homeland Security. The authors wish to thank Tampa Fire Rescue for its assistance in organizing and implementing this study.

\section{Conflict of interest}

The authors declare that they have no conflict of interest.

\section{References}

[1] International Association of Fire Fighters and International Association of Fire Chiefs. The Fire Service Joint Labor Management Wellness-Fitness Initiative, 3rd edition. Washington DC: International Association of Fire Fighters, 2008.

[2] Nuwayhid I, Stewart W, Johnson J. Work activities and the onset of first-time low back pain among New York City fire fighters. Am J Epidemiol. 1993;137(5):539-48.

[3] Gentzler M, Stader S. Posture stress on firefighters and emergency medical technicians (EMTs) associated with repetitive reaching, bending, lifting, and pulling tasks. Work. 2010;37(3):227-39.

[4] Butler RJ, Contreras M, Burton LC, Plisky PJ, Goode A, Kiesel K. Modifiable risk factors predict injuries in firefighters during training academies. Work. 2013;46(11-7).

[5] Beneck GJ, Kulig K. Multifidus atrophy is localized and bilateral in active persons with chronic unilateral low back pain. Arch Phys Med Rehabil. 2012;93(2):300-6.

[6] Hides JA, Gilmore C, Stanton WR, Bohlscheid E. Multifidus size and symmetry among chronic LBP and healthy asymptomatic subjects. Man Ther. 2008;13(1):43-9.

[7] Hides JA, Stokes MJ, Saide M, Jull GA, Cooper DH. Evidence of lumbar multifidus muscle wasting ipsilateral to symptoms in patients with acute/subacute low back pain. Spine. 1994;19(2):165-72.

[8] Akbari A, Khorashadizadeh S, Abdi G. The effect of motor control exercise versus general exercise on lumbar local stabilizing muscles thickness: Randomized controlled trial of patients with chronic low back pain. J Back Musculoskelet Rehabil. 2008;21:105-12.

[9] Choi G, Raiturker PP, Kim MJ, Chung DJ, Chae YS, Lee SH. The effect of early isolated lumbar extension exercise pro- gram for patients with herniated disc undergoing lumbar discectomy. Neurosurgery. 2005;57(4):764-72; discussion -72.

[10] Danneels LA, Vanderstraeten GG, Cambier DC, Witvrouw EE, Bourgois J, Dankaerts W, et al. Effects of three different training modalities on the cross sectional area of the lumbar multifidus muscle in patients with chronic low back pain. $\mathrm{Br}$ J Sports Med. 2001;35(3):186-91.

[11] Hides JA, Stanton WR, McMahon S, Sims K, Richardson CA. Effect of stabilization training on multifidus muscle crosssectional area among young elite cricketers with low back pain. J Orthop Sports Phys Ther. 2008;38(3):101-8.

[12] Kim J, Gong W, Hwang B. The effects of resistivity and stability-combined exercise for lumbar muscles on strength, cross-sectional area and balance ability: Exercises for prevention of lower back pain. J Phys Ther Sci. 2011;23(2):247.

[13] Storheim K, Holm I, Gunderson R, Brox J, B?K. The effect of comprehensive group training on cross-sectional area, density, and strength of paraspinal muscles in patients sicklisted for subacute low back pain. J Spinal Disord Tech. 2003;16(3):271-9.

[14] Teyhen DS, Childs JD, Stokes MJ, Wright AC, Dugan JL, George SZ. Abdominal and lumbar muscle size and symmetry at rest and during contracted states. normative reference ranges. J Ultrasound Med. 2012;31(7):1099-110.

[15] American College of Sports Medicine. ACSM's guidelines for exercise testing and prescription. 8th ed. Thompson W, editor. New York: Lippincott Williams \& Wilkins; 2010.

[16] Mayer JM, Mooney V, Matheson LN, Erasala GN, Verna JL, Udermann BE, et al. Continuous low-level heat wrap therapy for the prevention and early phase treatment of delayed onset muscle soreness of the low back: A randomized controlled trial. Arch Phys Med Rehabil. 2006;87(10):1310-7.

[17] Roland M, Fairbank J. The Roland-Morris Disability Questionnaire and the Oswestry Disability Questionnaire. Spine. 2000;25(24):3115-24.

[18] Shephard RJ. PAR-Q, Canadian home fitness test, and exercise screening alternatives. Sports Med. 1988;5(3):1985-95.

[19] Atlas SJ, Deyo RA. Evaluating and managing acute low back pain in the primary care setting. J Gen Intern Med. $2001 \mathrm{Feb}$; 16(2):120-31.

[20] Mayer JM, Graves JE, Verna JL, Pierra EA, Robertson VL, Ploutz-Snyder LL. Electromyographic activity of the lumbar extensor muscles: Effect of angle and hand position during Roman chair exercise. Arch Phys Med Rehabil. 1999;80(7):751-5.

[21] McGill SM. Low back exercises: Evidence for improving exercise regimens. Phys Ther. 1998;78(7):754-65.

[22] Verna JL, Mayer JM, Mooney V, Pierra EA, Robertson VL, Graves JE. Back extension endurance and strength: The effect of variable-angle roman chair exercise training. Spine. 2002;27(16):1772-7.

[23] Mayer JM, Verna JL, Manini T, Mooney V, Graves JE. Electromyographic activity of the trunk extensor muscles: Effect of varying hip position and lumbar posture during Roman chair exercise. Arch Phys Med Rehabil. 2002;83(11):1543-6.

[24] Stokes M, Rankin G, Newham D. Ultrasound imaging of lumbar multifidus muscle: Normal reference ranges for measurements and practical guidance on the technique. Man Ther. 2005;10:116-26.

[25] Hides JA, Richardson CA, Jull GA. Magnetic resonance imaging and ultrasonsgraphy of the lumbar multifidus muscle. Spine. 1995;20(1):54-8. 